\title{
Picosecond to microsecond TR-XAS: intermediates in the photolysis of methylCobalamin
}

Ganesh Subramanian $^{1}$, Gerdenis Kodis ${ }^{2}$, Andrew Chizmeshya ${ }^{2}$, Qingyu Kong ${ }^{3}$, Xiaoyi Zhang ${ }^{3}$, Uwe Weierstall ${ }^{1}$, John Spence ${ }^{1}$

${ }^{1}$ Department Of Physics, Arizona State University, Tempe, United States, ${ }^{2}$ School of Molecular Sciences, Arizona State University, Tempe, United States, ${ }^{3}$ Structural Science, X-ray Science Division, Advanced Photon Source, Argonne National Laboratory, Lemont, United States

E-mail: gsubram5@asu.edu

Macrocycle based catalysts utilize their axial sites to engage in catalysis. The biologically active cobalamins follow the same mechanism wherein cleavage of the Cobalt-Carbon (Co-C) bond is the most crucial step in the catalysis. In mammals, Methylcobalamin (MeCbl) plays a crucial role as a coenzyme of methionine synthase, which is required for the formation of methionine from homocysteine in the methylation cycle that involves DNA or proteins. Insufficient amounts of MeCbl could result in the development of megaloblastic anemia and eventually sub-acute combined degeneration of the spinal cord [1]. Consequently, cobalamins are rigorously tested as potential therapeutic drugs: promoting the regeneration of injured nerves, treating/slowing cognitive decline arising from Alzheimer's disease etc.

In understanding the catalytic activity of MeCbl, a variety of experiments have been performed. Under non-enzymatic conditions, the Co-C bond cleavage is generally achieved by using optical photons as with transient optical absorption [2]. $\mathrm{Ab}$-initio simulations of the MeCbl electronic structure [3] have also been performed, in conjunction with these experiments. Yet, due to lack of atomic resolution site-specific measurements, there is no conclusive evidence on the intermediate structures and the reaction pathways.

In this work, we report the first ever time-resolved X-ray measurements performed to study the photolysis of methylcobalamin at the beamline 11-ID-D, at the Advanced Photon Source (APS). The experiments involve an optical laser excitation at $400 \mathrm{~nm}$ to initiate the reaction followed by measuring the X-ray absorption spectra about the Cobalt Ka edge in fluorescence mode. Core-shell spectroscopy is used as the preferred technique since it can provide signatures of the element specific oxidation state and coordination. With the pump and probe scheme, the absorption spectra were collected across timescales, from picoseconds to microseconds, and two stable intermediate signatures were identified. Molecular and electronic structures for the reactant, intermediates and product were simulated by the density functional method (DFT) using the Gaussian 09 program (including explicit models and TD-DFT generated electronic excited states). These calculations were performed using allocations from the Arizona State University Advanced Computing Center (A2C2). Subsequently the structures were fed to the FEFF8.4 package to simulate for the X-ray absorption spectra as comparison to the experimental measurements. Simultaneous calculations were also carried out using the ORCA program to simulate the pre-edge features of the absorption spectra for all the models. The experimental data were processed and averaged using the DEMETER package (ATHENA and ARTEMIS). This project is supported by the STC Program of the National Science Foundation through BioXFEL under Agreement No. 1231306.

1. Banerjee, Ruma V., and Matthews, Rowena G. (1990). The FASEB journal 4.5, 1450-1459.

2. Walker, Larry A., et al. (1998). Journal of the American Chemical Society 120.15, 3597-3603.

3. Lodowski, Piotr, et al. (2014). The Journal of Physical Chemistry A 118.50 11718-11734.

Keywords: $\mathrm{X}$-ray absorption spectroscopy, time-resolved, Vitamin B12 